\title{
Title: Revisiting the basis for haemoglobin screening in pregnancy
}

\section{Authors}

Jahnavi Daru (1)

Soha Sobhy (1)

Sue Pavord (2)

\section{Affiliations}

1. Women's Health Research Unit, Centre for Primary Care and Public Health, Yvonne Carter Building, E1 2AB

2. Oxford University Hospitals NHS Foundation Trust

\section{Corresponding author}

Jahnavi Daru

Address:

Centre for Primary Care and Public Health

Yvonne Carter Building

58 Turner Street

London

E1 2AB

Telephone number: +44 2078826309

Email address: j.daru@qmul.ac.uk 
Abstract (200 words max.):

Purpose of review:

Anaemia affects up to $50 \%$ of pregnancies worldwide and is associated with maternal and neonatal morbidity and mortality. Prevention and management of anaemia remains a priority. Despite this, there is ongoing debate on the optimal approach to identifying anaemia in pregnant women and the best strategies for prevention and management. The objective of this review is to describe the current landscape of haemoglobin testing in pregnancy in low and high income countries.

\section{Recent findings:}

Current definitions of anaemia in pregnancy comprise a laboratory threshold of haemoglobin below which treatment is offered. Haemoglobin measurement is not sensitive in detecting iron deficiency, the most common cause of maternal anaemia. Furthermore, these historical thresholds were derived from heterogeneous populations comprising men and women. Women with anaemia in pregnancy are offered iron therapy, without testing for the underlying cause. This may be appropriate in high income settings, where iron deficiency is the likely cause but may not address the complex causes of anaemia in other geographical areas.

\section{Summary:}

Current thresholds of haemoglobin defining anaemia in pregnancy are under review. Further research and policy should focus on optimal strategies to identify women at risk of anaemia from all causes. 


\title{
Key words
}

Haemoglobin

Anaemia

Prevention

\begin{abstract}
Abbreviations
LMIC: low and middle-income country

LLINs: long-lasting insecticidal nets LLINs

IPTP: intermittent preventive treatment in pregnancy

WHO: World Health Organization
\end{abstract}




\section{Introduction}

Testing for anaemia in pregnancy is ubiquitous across low and high income countries and usually begins at the booking (first antenatal) appointment. Screening may range from looking for risk factors and clinical features of anaemia in the history, to point of care testing or laboratory assessment of the full blood count with or without assessment of iron parameters from a venous sample. (1) Which of these provides the most reliable and cost effective assessment remains unanswered. In this review, we will provide an overview of the landscape of the literature surrounding the use of haemoglobin as a screening tool for anaemia in pregnancy, the implications this has on clinical care, resource allocation and national and international policy.

\section{Why is anaemia screening important?}

It is widely acknowledged that anaemia in pregnancy is associated with significant morbidity and mortality (if severe) for the mother, independently of other factors. (2) Anaemia, if combined with other risks (such as emergency delivery) increases adverse outcomes multifold. (3)

Anaemia has also been associated with adverse outcomes for the developing baby, including low birth weight and preterm delivery. (4) This relationship has been demonstrated to be non-linear (5) meaning low and high values of haemoglobin are both detrimental to fetal growth and health. This calls into question the safety of unselected routine iron supplementation without testing for anaemia or iron deficiency.

\section{High-income countries}


The British Society for Haematology (BSH), RCOG and NICE guidelines recommend routine testing for anaemia at 12 and 28 weeks of pregnancy. (6) UK guidelines stress the importance of early identification and management of iron deficiency. As iron deficiency is the most common cause of maternal anaemia in high income settings, the UK guidelines recommend initiating oral iron supplements empirically to anaemic women, and using haemoglobin response as a diagnostic aid. This reduces the need for additional screening tests and avoids delays in management.

These strategies may miss non-anaemic iron depleted women if attention is not paid to those who may be at risk. (7) One study showed $42 \%$ of non-anaemic women in the first trimester had a marker of iron deficiency. (8) This may make a case for routine iron supplementation. However, in 2015, a review by the U.S. Preventive Services Task Force concluded that, while routine supplementation improved haematological parameters, there was insufficient evidence of improved maternal and infant outcomes. This is an area of ongoing debate, with recent rebuttals suggesting that routine iron supplementation does indeed improve clinical outcomes, (8) however, trial level data are lacking. Currently, routine administration of iron interventions to all pregnant women in high income settings is not recommended. (7)

The ongoing high prevalence of anaemia globally and the lack of evidence demonstrating safety and efficacy of routine iron supplementation in pregnancy highlights unmet clinical and research needs.

\section{Low and middle income countries}

More than 1.6 billion people are anaemic, with $42 \%$ of all pregnant women worldwide affected. (9) The prevalence and severity of anaemia is highest in low and middle-income countries and is a public health concern, with the highest rates and mortality associated with 
the condition being found in South and South East Asia, and Sub-Saharan Africa. (10) In these settings the causes of anaemia are often complex, however the global focus currently remains on iron deficiency anaemia. (11) Although iron deficiency is thought to be the major cause of anaemia, thought to account for around $50 \%$ of anaemia, there is little evidence to support such estimates,(12) with a fall in haemoglobin attributed to iron deficiency, without objective testing. (13) The limitations of existing indicators to measure iron status at a population level and scarcity of data prevent clear understanding of the relative contribution of iron deficiency to anaemia prevalence estimates in different geographical settings. Although some anaemia prevention strategies have proven successful, (14) anaemia continues to pose a considerable public health burden, particularly in pregnancy. (15) Better understanding of the causes of anaemia, testing methods and treatment of the underlying cause may go a long way to reducing the associated burden. A more comprehensive and holistic approach then just iron supplementation is needed.

The World Health Organization also recommends that all pregnant women are routinely screened for anaemia. (1) Those found to be anaemic should be offered daily iron supplementation and those not yet anaemic offered iron and folic acid weekly. (1) The effectiveness of this strategy in reducing rates of anaemia worldwide is unclear. Particularly within LMICs, where anaemia has many potential causes and contributors besides iron deficiency, including genetic causes, inflammation, infections both viral and parasitic; such as helminth, malaria and HIV and malnutrition and micronutrient deficiencies. Below we discuss a few key issues briefly. 
Poor diet and food scarcity in vulnerable groups, including pregnant women worsen nutritional anemias including those such as iron, B12 and folate, all of which cause a fall in haemoglobin. (16) While each micronutrients have specific roles, multiple deficiencies tend to cluster within individuals, acting synergistically increasing the burden of disease. In these individuals, iron supplementation alone is unlikely to lead to anaemia resolution.

\section{Soil-transmitted helminths}

Infectious diseases such as hookworm reduce absorption and metabolism of key micronutrients (notably iron) in addition to increasing gastrointestinal tract losses. Hookworm infections contribute greatly to the high prevalence of anaemia in sub-Saharan Africa and Southeast Asia, with an estimated 576-740 million infections. (17) There are clear benefits from pooled estimates of randomized controlled trials of deworming and concomitant iron supplementation strategies showing improvements in mean haemoglobin concentration across sub-Saharan Africa. (18) As a result, the current WHO recommendation for preventive chemotherapy (deworming) include using single-dose albendazole (400 mg) or mebendazole $(500 \mathrm{mg}$ ), as a public health intervention for pregnant women, after the first trimester, living in areas where both anaemia and soil transmitted helminth infections are considered endemic. (19)

\section{Malaria}

Malarial infection, particularly plasmodium falciparum can lead to and worsen preexisting anaemia.(20) The balance of iron supplementation in the context of malarial infection (suspected or confirmed) requires careful consideration; it has been an area of considerable debate. $(20,21)$ In areas such as Africa, WHO recommends prevention and treatment of malaria during pregnancy using of long-lasting insecticidal nets (LLINs); in all areas with moderate to high malaria transmission in Africa, intermittent preventive treatment in 
pregnancy (IPTP) with sulfadoxine-pyrimethamine (SP), as part of antenatal care services and prompt diagnosis and effective treatment of malaria infections.(1)

\section{HIV related causes}

HIV infection and anaemia are closely related, with anaemia being both a common complication in addition to being a marker of disease progression. $(22,23)$ HIV/AIDS-related anaemia is more common in sub-Saharan Africa. The mechanism of HIV/AIDS-related anaemia is complex, and beyond the scope of this review. Anaemia is known directly result from HIV infection and anaemia of chronic disease, and indirectly affected by antiretroviral therapy and AIDS-related illnesses. (23)

\section{Genetic causes}

Haemoglobin disorders affect populations in LMICs disproportionately more than other areas of the world, particularly Africa and South East Asian regions where $18.2 \%$ and $6.6 \%$ of the population, respectively, carry a significant haemoglobin variant, as a result affecting more than $7 \%$ of pregnant women worldwide. (24) Although a known cause of anaemia, their contribution to the global anaemia burden is unclear. The safety of iron supplementation in pregnant women with haemoglobinopathies, particularly at high doses is unclear, (25) although some limited data suggest iron overload is unlikely to occur. (26)

Distinguishing the underlying causes of anaemia requires clinical observation and expensive laboratory investigation, beyond the scope of many regions in LMIC settings.

\section{The ongoing debate}


Haemoglobin, as a test is reported along a continuous scale. The current definitions of anaemia are based on laboratory values taken from a healthy population where two standard deviations below the lower limit of normal distribution are considered abnormal (classically $105 \mathrm{~g} / \mathrm{L}$ in pregnancy, to account for plasma volume dilution). Values of haemoglobin below this disease defining threshold necessitate treatment, but cannot suggest what treatment should be offered, as a fall in haemoglobin is multifactorial. Consequently, the diseasedefining threshold for anaemia, particularly in pregnancy, needs to be considered with caution. There is increasing awareness that the evidence base behind widely used thresholds for anaemia and iron biomarkers in pregnancy is poor. $(27,28)$ Moreover, the reference ranges of haemoglobin determining anaemia in pregnancy were developed using data from unselected cohorts of individuals including men and non-pregnant women. $(29,30)$

Many argue the widely used thresholds for anaemia in pregnancy are not accurate, (31) particularly as these historical reference ranges did not account for geographical or ethnic variation, in addition to the burden of infectious disease which undoubtedly will influence the distribution of laboratory blood values in any population and especially pregnant women. (32, 33)

This leads to the question: are the strategies we are using for identifying anaemic women fit for purpose? Perhaps not. Currently there is ongoing work by WHO to look at the evidence behind anaemia defining haemoglobin levels used in clinical practice and research, which may change the tide in this area. $(31,34)$

\section{What does this mean for clinicians?}

A threshold based approach to make treatment decision is longstanding in numerous areas of medicine. (35) However, changing disease defining thresholds (for example if anaemia was 
defined as a haemoglobin level $<120 \mathrm{~g} / \mathrm{L}$ rather than 110 ), will have profound consequences on numerous aspects of care. For example, having anaemia precludes blood donation, a vital resource with limited availability worldwide. (36) New definitions of anaemia (especially if the thresholds are increased) will have a profound effect on the number of eligible donors and consequently blood stores available, particularly in emergency settings such as obstetric haemorrhage.

If a consensus on the definition of anaemia is currently in a state of flux, what does this mean for determining the effectiveness of treatment? Most randomised controlled trials of iron treatments in pregnancy have a change in haemoglobin, or prevention of anaemia and avoidance of blood transfusion as primary outcomes. (37) The findings of these studies in turn drive policy and clinical guidelines. As a consequence, guidelines and policy may be frustratingly vague. Until these issues are resolved, it is important for clinicians to consider that individual pregnant women will experience anaemia related symptoms, even if their blood parameters do not suggest overt anaemia. Treatment decisions for these women must be made on more than blood values alone. It is also important to recognise that causes of anaemia beyond iron deficiency should be considered and providing iron interventions is not sufficient to solve the problem of anaemia.

\section{What next?}

There are emerging Australian data suggesting testing and treatment of non-anaemic iron deficiency would be beneficial in reducing transfusion post-delivery, however this has not been tested in trial settings. (38) 
There are limited data on the use of other laboratory tests as screening tools. In routine clinical practice a low haemoglobin, mean cell volume (MCV) and mean cell haemoglobin $(\mathrm{MCH})$, and mean cell haemoglobin concentration $(\mathrm{MCHC})$ are suggestive of iron deficiency, but need cautious interpretation. A recently published study in Sri Lanka found MCHC to be sensitive marker for iron deficiency, performing well compared to haemoglobin and MCV. However, further testing and cost effectiveness analyses would be required before this could be implemented outside of research. (39)

Testing for specific causes of anaemia, for example markers of iron status such as, but not limited to serum ferritin and transferrin levels are fraught with complexity in pregnancy, due to the physiological changes.(40) Normal ranges for these markers in pregnancy have not been validated and the currently used thresholds of serum ferritin to diagnosed iron deficiency are based on less than ideal data. (28)

Other markers such as serum hepcidin (a key homeostatic regulator of iron metabolism) are currently being tested in pregnancy settings, but are not routinely available for clinical use.

\section{Conclusion}

Haemoglobin is still widely used as a screening tool for anaemia in pregnancy. In the UK, a fall in haemoglobin is most commonly as a result of iron deficiency and so current guidelines recommend oral iron therapy as first line management. In LMIC settings, iron supplementation alone is unlikely to improve anaemia prevalence.

\section{Word count: 2,009 (exc references)}




\section{Key points}

- A haemoglobin level below a particular threshold defines anaemia. Currently used thresholds are based on poor evidence and are currently under review

- Anaemia is caused by a variety of factors, the most common of which is a deficiency of iron. Treatment for anaemia with iron interventions without testing for the underlying cause is widespread

- The application of other laboratory tests in pregnancy is currently limited.

\section{Acknowledgements}

JD has received fees from Pharmacosmos as part of an advisory board assessing the adverse effects of intravenous iron. SP has received lecture fees from Pharmacosmos and Vifor. Both JD and SP are authors of the UK guideline on iron deficiency anaemia in pregnancy. SS has no conflicts of interest to declare.

\section{Funding: none}




\section{References}

1. World Health Organization. WHO recommendations on antenatal care for a positive pregnancy experience. Geneva, Switzerland: World Health Organization, Research RH; 2016 7th November 2016.

2. * Daru J, Zamora J, Fernández-Félix BM, Vogel J, Oladapo OT, Morisaki N, et al. Risk of maternal mortality in women with severe anaemia during pregnancy and post partum: a multilevel analysis. The Lancet Global Health. 2018;6(5):e548-e54.

This was the first study to show an independent relationship between severe anaemia and maternal mortality when all other factors, including postpartum haemorrhage are accounted for, using an international database.

3. Sobhy S, Arroyo-Manzano D, Murugesu N, Karthikeyan G, Kumar V, Kaur I, et al. Maternal and perinatal mortality and complications associated with caesarean section in lowincome and middle-income countries: a systematic review and meta-analysis. Lancet. 2019;393(10184):1973-82.

4. Scanlon KS, Yip R, Schieve LA, Cogswell ME. High and low hemoglobin levels during pregnancy: differential risks for preterm birth and small for gestational age. Obstetrics \& Gynecology. 2000;96(5, Part 1):741-8.

5. Jung J, Rahman MM, Rahman MS, Swe KT, Islam MR, Rahman MO, et al. Effects of hemoglobin levels during pregnancy on adverse maternal and infant outcomes: a systematic review and meta-analysis. Annals of the New York Academy of Sciences. 2019.

6. Pavord S, Myers B, Robinson S, Allard S, Strong J, Oppenheimer C. UK guidelines on the management of iron deficiency in pregnancy. British Journal of Haematology. 2012;156(5):588-600. 
7. ** Pavord S., Daru, J., Prasannan, N., Stanworth, SJ., Robinson, SE., Girling, J. UK guideline for the management of iron deficiency in pregnancy. British Journal of Haematology. 2019; [in press]

This is the most recent guideline on the prevention and management of iron deficency anaemia in pregnancy produced by the British Society for Haematology.

8. * Auerbach M, Abernathy J, Juul S, Short V, Derman R. Prevalence of iron deficiency in first trimester, nonanemic pregnant women. The Journal of Maternal-Fetal \& Neonatal Medicine. 2019:1-4.

This is the first study to demonstrate the prevalance of non anaemic iron defciency and how treatment of this may improve clinical outcomes for pregnant women.

9. Benoist Bd, McLean E, Egll I, Cogswell M. Worldwide prevalence of anaemia 19932005: WHO global database on anaemia: World Health Organization; 2008.

10. Pasricha S-R. Anaemia in pregnancy-not just iron deficiency. Acta haematologica. 2013;130(4):279-80.

11. World Health Organization.. Guideline: intermittent iron and folic acid supplementation in non-anaemic pregnant women. 2011.

12. Thurlow RA, Winichagoon P, Green T, Wasantwisut E, Pongcharoen T, Bailey KB, et al. Only a small proportion of anemia in northeast Thai schoolchildren is associated with iron deficiency-. The American journal of clinical nutrition. 2005;82(2):380-7.

13. Lim SS, Vos T, Flaxman AD, Danaei G, Shibuya K, Adair-Rohani H, et al. A comparative risk assessment of burden of disease and injury attributable to 67 risk factors and risk factor clusters in 21 regions, 1990-2010: a systematic analysis for the Global Burden of Disease Study 2010. The lancet. 2012;380(9859):2224-60. 
14. Casey GJ, Sartori D, Horton SE, Phuc TQ, Phu LB, Thach DT, et al. Weekly ironfolic acid supplementation with regular deworming is cost-effective in preventing anaemia in women of reproductive age in Vietnam. PloS one. 2011;6(9):e23723.

15. ** Stevens GA, Finucane MM, De-Regil LM, Paciorek CJ, Flaxman SR, Branca F, et al. Global, regional, and national trends in haemoglobin concentration and prevalence of total and severe anaemia in children and pregnant and non-pregnant women for 1995-2011: a systematic analysis of population-representative data. The Lancet Global health. 2013;1(1):e16-e25.

This is the most comprehensive assessment of anaemia prevalance ratesin a global population, including among pregnant women.

16. Balarajan Y, Ramakrishnan U, Ozaltin E, Shankar AH, Subramanian SV. Anaemia in low-income and middle-income countries. The Lancet. 2011;378(9809):2123-35.

17. Bethony J, Brooker S, Albonico M, Geiger SM, Loukas A, Diemert D, et al. Soiltransmitted helminth infections: ascariasis, trichuriasis, and hookworm. The lancet. 2006;367(9521):1521-32.

18. Gulani A, Nagpal J, Osmond C, Sachdev HPS. Effect of administration of intestinal anthelmintic drugs on haemoglobin: systematic review of randomised controlled trials. BMJ. 2007;334(7603):1095.

19. World Health Organization. Guideline: preventive chemotherapy to control soiltransmitted helminth infections in at-risk population groups: World Health Organization; 2017.

20. Prentice AM. Iron Metabolism, Malaria, and Other Infections: What Is All the Fuss About? The Journal of Nutrition. 2008;138(12):2537-41.

21. Gera T, Sachdev H. Effect of iron supplementation on incidence of infectious illness in children: systematic review. BMJ. 2002;325(7373):1142. 
22. May M, Boulle A, Phiri S, Messou E, Myer L, Wood R, et al. Prognosis of patients with HIV-1 infection starting antiretroviral therapy in sub-Saharan Africa: a collaborative analysis of scale-up programmes. The Lancet. 2010;376(9739):449-57.

23. Volberding PA, Levine AM, Dieterich D, Mildvan D, Mitsuyasu R, Saag M, et al. Anemia in HIV infection: clinical impact and evidence-based management strategies.

Clinical infectious diseases. 2004;38(10):1454-63.

24. Modell B, Darlison M. Global epidemiology of haemoglobin disorders and derived service indicators. Bulletin of the World Health Organization. 2008;86:480-7.

25. Pasricha SR, Drakesmith H, Black J, Hipgrave D, Biggs BA. Control of iron deficiency anemia in low- and middle-income countries. Blood. 2013;121(14):2607-17. 26. Aroke D, Tchouakam DN, Kadia BM, Choukem SP. Iron supplementation in pregnant sicklers: an opinion. BMC Pregnancy Childbirth. 2018;18(1):256.

27. Roy NBA and Pavord S. The management of anaemia and haematinic deficiencies in pregnancy and post-partum. Transfusion Medicine. 2018;28(2):107-16.*

28. Daru J, Allotey J, Pena-Rosas JP, Khan KS. Serum ferritin thresholds for the diagnosis of iron deficiency in pregnancy: a systematic review. Transfusion medicine (Oxford, England). 2017;27(3):167-74.

29. Kilpatrick G. Prevalence of anaemia in the general population. British medical journal. 1961;2(5269):1736.

30. Natvig K. Studies on hemoglobin values in Norway. 5. Hemoglobin concentration and hematocrit in men aged 15-21 years. Acta Medica Scandinavica. 1966;180:613-20.

31. Ferguson M, Dennis A. Defining peri-operative anaemia in pregnant womenchallenging the status quo. Anaesthesia. 2019;74(2):237-45. 
32. Gonzales GF, Tapia V, Gasco M. Correcting haemoglobin cut-offs to define anaemia in high-altitude pregnant women in Peru reduces adverse perinatal outcomes. Archives of gynecology and obstetrics. 2014;290(1):65-74.

33. Wirth JP, Woodruff BA, Engle-Stone R, Namaste SM, Temple VJ, Petry N, et al. Predictors of anemia in women of reproductive age: Biomarkers Reflecting Inflammation and Nutritional Determinants of Anemia (BRINDA) project. The American Journal of Clinical Nutrition. 2017;106(suppl_1):416S-27S.

34. * Garcia-Casal MN, Pasricha SR, Sharma AJ, Pena-Rosas JP. Use and interpretation of hemoglobin concentrations for assessing anemia status in individuals and populations: results from a WHO technical meeting. Ann N Y Acad Sci. 2019.

This is a paper summarising the ongoing activity by WHO on defining anaemia in populations, including in pregnant women.

35. Giannoni A, Baruah R, Leong T, Rehman MB, Pastormerlo LE, Harrell FE, et al. Do optimal prognostic thresholds in continuous physiological variables really exist? Analysis of origin of apparent thresholds, with systematic review for peak oxygen consumption, ejection fraction and BNP. PLoS One. 2014;9(1):e81699.

36. Salvin HE, Pasricha SR, Marks DC, Speedy J. Iron deficiency in blood donors: a national cross-sectional study. Transfusion. 2014;54(10):2434-44.

37. Malinowski AK, D'Souza R, Khan KS, Shehata N, Malinowski M, Daru J. Reported Outcomes in Perinatal Iron Deficiency Anemia Trials: A Systematic Review. Gynecol Obstet Invest. 2019:1-18.

38. Crispin P, Stephens B, McArthur E, Sethna F. First trimester ferritin screening for pre-delivery anaemia as a patient blood management strategy. Transfusion and Apheresis Science. 2018. 
39. Rabindrakumar MSK, Pujitha Wickramasinghe V, Gooneratne L, Arambepola C, Senanayake H, Thoradeniya $\mathrm{T}$. The role of haematological indices in predicting early iron deficiency among pregnant women in an urban area of Sri Lanka. BMC Hematol. 2018;18:37.

40. Demmers MW, Niens M, van der Haar G, van der Zaag-Loonen HJ, Hoffmann JJ, Adriaansen HJ. Functional iron deficiency markers are absent during pregnancy despite evidence of low iron stores. Ann Clin Biochem. 2019;56(4):450-6.

41. Zaman B, Rasool S, Jasim S, Abdulah D. Hepcidin as a diagnostic biomarker of iron deficiency anemia during pregnancy. The Journal of Maternal-Fetal \& Neonatal Medicine. 2019:1-9. 